\section{What is IUGS?}

The International Union of Geological Sciences is one of the largest and most active scientific non-governmental organisations in the world. Founded in 1961, IUGS is a member of the International Council of Scientific Unions (ICSU). IUGS promotes and encourages the study of geological problems, especially those of world-wide significance, and supports and facilitates international, multidisciplinary cooperation and dialogue in the earth sciences.

The IUGS Commissions, Committees and Boards are concerned with a wide range of geological research of direct interest to governments, industry and academic institutions within the earth sciences. Major effort is directed towards international Joint Programmes sponsored by IUGS and other organisations, Affiliated organisations are large autonomous international associations which share with IUGS an interest in planning and undertaking activities and meetings of mutual benefit.

Membership of IUGS is open to countries generally through a designated 'adhering organisation' which may be a national committee or some other national earth science body or institution. Each adhering organisation pays an annual subscription according to its category of membership. A special class of membership (under four categories) has been established to enable industry and national and international bodies (both governmental and nongovernmental) as well as individuals, to belong to IUGS and to participate, to various degrees, in the planning of IUGS activities according to their category of membership.

The ultimate authority for the management of IUGS is vested in the Council which is composed of representatives from the national adhering organisations. It usually meets every four years during the International Geological Congress to review progress by IUGS constituent bodies, the administration of the Union and its accounts, and to decide on any new proposals or initiatives.

Overall management is the responsibility of the Executive Committee which is elected by Council on the recommendation of a Nominating Committee. The Executive Committee normally meets annually and deals with major policy, operational and administrative issues affecting the Union. Current members of the Executive Committee are listed on the inside front cover together with the adhering members. Further information about IUGS may be obtained from :

The IUGS Secretariat Geological Survey of Norway

PO Box 3006 Lade N-7002 Trondheim Norway

\section{COMMISSIONS}

\section{COMMISSION ON COMPARATIVE} PLANETOLOGY (CCP)

Dr. Peter Janle, Chairman

Institut fer Geophysik der Universitaet

Olshausenstr. 40-60, D-24098 Kiel GERMANY

Tel: +494318803902 ;

Fax: +494318804432

E-mail: geo23@physik.uni-kiel.d400.de

\section{COMMISSION ON FOSSIL FUELS (CFF)}

Dr. R. Sinding-Larsen, Chairman

Norwegian Institute of Technology,

Department of Geology, Høgskoleringen 6,

N-7034 Trondheim-NTH, NORWAY

Tel: +4773594837

Fax: +4773594814

COMMISSION ON GEOLOGICAL SCI-

ENCES FOR ENVIRONMENTAL PLANNING (COGEOENVIRONMENT)

Dr. Ed F.J. de Mulder, Chairman

Rijks Geologische Dienst, P.O. Box 157

2000 AD Haarlem, THE NETHERLANDS

Tel: +31235300292

Fax: + 31235262709

Telex: 71105 geold $\mathrm{nl}$

E-mail: demulder@worldaccess.n!

\section{COMMISSION ON GLOBAL SEDIMEN-}

\section{TARY GEOLOGY (GSGP)}

Dr. Benoit Beauchamp, Chairman

Geological Society of Canada

3303 33rd Street NW, Calgary

Alberta T2N 1 Y9, CANADA

Tel: (403) 292-7190

Fax: (403) 292-5377;

E-mail: bbeauchamp@gsc.emr.ca

\section{COMMISSION ON THE HISTORY OF}

\section{GEOLOGICAL SCIENCES (INHIGEO)}

Dr. Hugh S. Torrens, President

Department of Geology, The University, Keele,

Staffordshire ST5 5BG. UK

Tel: +44 1782583172 :

Fax: +441782715261

E-mail:GGA10@keele.ac.uk

COMMISSION ON IGNEOUS AND METAMORPHIC PETROGENESIS (CIMP)

Dr. Ian Campbell, Chairman

Research School of Earth Sciences

The Australian National University

GPO Box 4, Canberra 0200, AUSTRALIA

Tel: +6162494366

Fax: +6162493683

E-mail: Ian.Campbell@anu.edu.au

COMMISSION ON THE MANAGEMENT AND APPLICATION OF GEOSCIENCE INFORMATION (COGEOINFO)

Dr. James E. Biesecker, Chairman 40782 Woodside Place, Leesburg,

VA 20175 , USA

Tel: +17037776357

\section{COMMISSION ON STRATIGRAPHY (ICS)}

Professor J. Remane. Chairman

Université de Neuchâtel Institut de Géologie, 11 rue Emile-Argand, $\mathrm{CH}-2000$ Neuchâtel, SWITZERLAND

Tel: +4138256434 or 411380

Fax: +413825588

E-mail: Jurgen.Remane@geol.unine.ch
COMMISSION ON SYSTEMATICS IN PETROLOGY (CSP)

Prof. Jörg Keller, Chairman

Institut für Mineralogie, Petrologie und Geo-

chemie, Albert-Ludwigs-Universität

Albertstraße 23b, D-79104 Freiburg.

D.79104 Freiberg GERMANY

Tel: +497612036404

Fax: +497612036407

E-mail: jkeller@ruf.uni-freiburg.de

\section{COMMISSION ON TECTONICS}

\section{(COMTEC)}

Dr. Paul L. Hancock, Chairman

Department of Geology, University of Bristol,

Wills Memorial Building, Queens Road,

Bristol BS8 IRJ, UK

Fax: +44(0) 1179253385

E-mail: P.L.Hancock@bris.ac.uk

\section{JOINT PROGRAMS}

GEOLOGICAL APPLICATIONS OF REMOTE SENSING (GARS)

Dr. D. Bannert, Chairman

Head, Remote sensing Section,

Federal Institute for Geosciences and Natural

Resources (BGR)

Hanover, GERMANY

INTERNATIONAL GEOLOGICAL CORRELATION PROGRAMME (IGCP) Dr. F. Wolfgang Eder.

Director.Division of Earth Sciences.

UNESCO, SC/GEO, 1 rue Miollis,

F-75732 Paris, Cedex 15 FRANCE

Tel: $+331456841.15 ;$ Fax: +33 । 43061122

Telex: 204461 Paris

\section{INTER-UNION COMMISSION ON THE} LITHOSPHERE (ICL)

Prof. Dr. A.G. Green, President Institut für Geophysik, ETH - Hönggerberg, Zürich, CH-8093, SWITZERLAND Tel: +41 1633 2657; Fax: +41 16331065 ; E-mail: green@geo.phys.ethz.ch

\section{IUGS/UNESCO PROGRAM ON EARTH} PROCESSES IN GLOBAL CHANGE

Dr. Nicole Petit-Maire, Co-Chairman Lab. de Geologie du Quaternaire CNRS, Luminy, Case 907.

F-13288 Marseille Cedex 9. FRANCE Tel: $+3391269659:$ Fax: +3391266638

Dr. T. Partridge, Co-Chairman

Transvaal Museum

P.O. Box 413, Pretoria, SOUTH AFRICA

IUGS/UNESCO PROJECT ON MINERAI AND ENERGY DEPOSIT MODELLING Dr. Richard B. McCammom, Chairman U.S. Geological Survey, 954 National Center Reston, VA 20192, USA

Tel +17036486150 ; Fax: + 7036486383

Telex: 49614145 usgsut;

E-mail: mccammon@usgs.gov

\section{INTERNATIONAL GEOLOGICAL} CONGRESS

31st International Geological Congress Rio de Janiero, Brazil - August 6-17, 2000 Prof. Hernani Chaves

President, Preparatory Commission

Ave. Pasteur, 404

Urca: Cep 22290-204, Rio de Janiero, BRAZIL. Tel: +55 21295 5337: Fax: +55 2l 5423647 ;

E-mail: Hernani@uerj.br 


\section{ADVISORY BOARD}

ADVISORY BOARD FOR PUBLICATIONS (ABP)

Dr. W.G.E. Caldwell, Chairman

Department of Earth Sciences

$B$ and $G$ Building, Room 61

University of Western Ontario

London, Ontario N6A 5B8, CANADA

Tel: +1 5196613857 ; Fax: +1 5196613198

E-mail: gcaldwel@julian.uwo.ca

ADVISORY BOARD FOR RESEARCH

DEVELOPMENT (ABRD)

Vacancy.

AFFILIATED ORGANIZA. TIONS

AMERICAN ASSOCIATION OF PETRO-

LEUM GEOLOGISTS (AAPG)

Dr. Gary D Howell, Science Director and International Development Advisor

The American Association of Petroleum Geologists, PO Box 979, 1444 South Boulder,

Tulsa, OK 74101-0979, USA

AMERICAN GEOLOGICAL INSTITUTE (AGI)

Marcus E. Milling, Executive Director

4220 King Street, Alexandria, VA 22302-1502,

USA. Tel: (703) 379-2480; (703) 379-7563;

E-mail: agi@agiweb.org

AMERICAN GEOPHYSICAL UNION (AGU)

A. F. Spilhaus Jr., Executive Director

2000 Florida Avenue. NW

Washington, D.C. 20009-1277, USA.

Tel: (202) 462-6900: Fax: (202) 328-0566

ASSOCIATION OF ARAB GEOLOGISTS (AGA)

Dr. Wissam S. Al-Hashami, Secretary General,

PO Box 1247, Baghdad 12112, IRAQ

ASSOCIATION OF EUROPEAN GEOLOGI-

\section{CAL SOCIETIES (AEGS)}

Dr. B. Deffontaines, Secretary

Société Géologique de France T26.16-1E-

Boite 129, Dept. Geotectonics-LGGST, 4

Place Jussieu, F-75252 Paris Cedex 05,

FRANCE

\section{ASSOCIATION OF EXPLORATION GEO-}

CHEMISTS (AEG)

Dr. Sherman P. Marsh, Secretary

US Geological Survey, Federal Center, MS 973,

Denver, CO 80225, USA

ASSOCIATION OF GEOSCIENTISTS FOR

INTERNATIONAL DEVELOPMENT

(AGID)

AGID Headquarters, Institute of Geosciences,

University of São Paulo, CP 20.899,

01498-970 São Paulo, BRAZIL

ASSOCIATION INTERNATIONALE POUR

L'ÉTUDE DES ARGILES (AIPEA)

Professor R. Schoonheydt, Secretary General

COK Katholieke Universiteit Leuven, Kardinaal

Mercierlaan 92. B-3001 Heverlee, BELGIUM

CARPATHIAN BALKAN GEOLOGICAL ASSOCIATION (CBGA)

Dr. Kristina Kolceva, Secretary

Faculty of Geology and Geography, Sohia University, 1504 Sofia, BULGARIA

CIRCUM-PACIFIC COUNCIL FOR ENERGY AND MINERAL RESOURCES

Dr. Michel T. Halbouty, President \& Chairman The Halbouty Center, 5100 Westheimer Road,

Houston, TX 77056, USA
COMMISSION FOR THE GEOLOGICAL MAP OF THE WORLD (CGMW)

Dr. Phillipe Bouysse, Secretary General

Maison de la Géologie, 77, rue Claude Bernard.

F-75005 Paris, FRANCE

EUROPEAN ASSOCIATION OF SCIENCE EDITORS (EASE)

Ms. Maeve O'Connor, Secretary Treasurer 49 Rossendale Way, London NW1 OXB, UK

GEOCHEMICAL SOCIETY (GS)

Dr. Steven B. Shirey, Secretary

The Geochemical Society, Department of Terrestrial Magnetism, Carnegie Institution of Washington, 5241 Broad Branch Road NW,

Washington, DC 20015, USA

GEOLOGICAL SOCIETY OF AFRICA (GSAF)

Dr. S. N. Ayonge, Secretary General, Geological Society of Africa, Department of Geology. University of Buea, PO Box 63, Buea, Cameroon

GEOLOGICAL SOCIETY OF AMERICA (GSAm)

Dr. Donald Davidson, Executive Director Geological Society of America, PO Box 9140 , Boulder, CO 80301, USA

\section{INTERNATIONAL ASSOCIATION OF} ENGINEERING GEOLOGY (IAEG) Dr. L. Primel, Secretary General Laboratoire Central des Ponts et Chaussess, 58 Boulevard Lefebvre, F-75732 Paris Cedex 15, FRANCE

INTERNATIONAL ASSOCIATION ON THE GENESIS OF ORE DEPOSITS (IAGOD) Ing. Jaroslav Aichler, Secretary General Czech Geological Survey, PO Box 6579001 Jesenik, CZECH REPUBLIC

INTERNATIONAL ASSOCIATION OF GEOCHEMISTRY AND COSMOCHEMISTRY (IAGC)

Dr. Mel Gascogne, Secretary

AECL Research, Whiteshell Laboratories,

Pinawa, Manitoba RoE 1LO, CANADA

INTERNATIONAL ASSOCIATION OF GEOMORPHOLOGISTS

Dr. Robert J. Allison, Secretary General Department of Geography, University of Durham, South Road, Durham DHI 3LE, UK

INTERNATIONAL ASSOCIATION OF HYDROGEOLOGISTS (IAH) Dr. A. Skinner, Secretary General, PO Box 9 Kenilworth, Warwickshire CV8 1JG, UK

INTERNATIONAL ASSOCIATION FOR MATHEMATICAL GEOLOGY (IAMG) Dr. Michael Ed Hohn, Secretary West Virginia Geological Survey, PO Box 879 , Morgantown, WV 26505, USA

INTERNATIONAL ASSOCIATION OF SEDIMENTOLOGISTS (IAS)

Dr. F. Surlyk, Secretary General

Geologisk Centralinstitut, Øster Voldgade 10, DK-1350 Copenhagen, DENMARK

\section{INTERNATIONAL ASSOCIATION OF} STRUCTURAL/TECTONIC GEOLOGISTS Dr. Sue H. Treagus, Convenor Department of Geology, University of Manchester, Manchester M13 9PL, UK

INTERNATIONAL CENTER FOR TRAINING AND EXCHANGES IN GEOSCIENCES (CIFEG)

Dr. Jean-Claude Napias, Director International Center for Training and Exchanges in the Geosciences, Avenue de Concyr, BP
6517, 45065 Orléans Cedex 2, FRANCE

INTERNATIONAL FEDERATION OF PALYNOLOGICAL SOCIETIES (IFPS)

Dr. Owen K. Davis, Secretary Treasurer Department of Geoscience, University of Arizona, Tucson, Arizona 85721, USA

INTERNATIONAL MINERALOGICAL ASSOCLATION (IMA)

Dr. S. S. Hafner, Secretary

Institute of Mineralogy, University of Marburg, Hans-Meerwein-Straße, D-35032 Marburg, GERMANY

INTERNATIONAL PALAEONTOLOGICAL ASSOCIATION (IPA)

Professor D. L. Bruton, Secretary General Universitet i Oslo, Sarsgate 1, N-0562 Oslo, NORWAY

INTERNATIONAL PERMAFROST ASSOCIATION

Dr. Jerry Brown, Secretary General PO Box 9200, Arlington, VA 22219-0200, USA

INTERNATIONAL SOCIETY FOR ROCK MECHANICS (ISRM)

Dr. Jose Delgado Rodrigues, Secretary General Laboratório Nacional de Engenharia Civil 101 101 Avenida do Brasil, P-1799 Lisboa Codex PORTUGAL

INTERNATIONAL SOCIETY FOR SOIL MECHANICS AND FOUNDATION ENGINEERING

Dr. R.H.G. Parry, Secretary General University Engineering Department, Trumpington Street, Cambridge CB2 IPZ, UK

INTERNATIONAL UNION FOR OUATERNARY RESEARCH (INQUA)

Dr. Edward Derbyshire, Secretary

Department of Geography, Royal Holloway, University of London, Egham, Surrey TW20 OEX, UK

THE METEORITICAL SOCIETY (MS)

Dr. Monica M. Grady, Secretary

Natural History Museum, Cromwell Road

London SW7 5BD, UK

SOCIETY OF ECONOMIC GEOLOGISTS (SEG)

John A. Thoms, Executive Secretary

5808 South Rapp Street. Suite 209.

Little Creek One Building, Littleton,

CO 80120-1942, USA

SOCIETY FOR GEOLOGY APPLIED TO MINERAL DEPOSITS (SGA)

Dr Maurice Pagel, Executive Secretary

CREGU BP-23, F-54501 Vandouvre les Nancy, FRANCE

SOCIETY FOR SEDIMENTARY GEOLOGY (SEPM)

Cathleen P. Williams, Executive Director 1731 E. 71 st Street, Tulsa, OK 74136, USA Tel: (800) 865-9765 (in North America) and (918) 493-3361 (Worldwide);

Fax: (918) 493-2093 